19

\title{
The structured development method SENN for feedforward neural networks and the application to the multimedia patient record in the Bavarian Health Net
}

\author{
Rolf Engelbrecht, Thomas Waschulzik ${ }^{1)}$, Wilfried Brauer ${ }^{2}$, Matthias \\ Scherf \\ GSF-Forschungszentrum, Medis-Institut, Neuherberg; ${ }^{1)}$ TZI \\ Intelligente Systeme, Universität Bremen; ${ }^{2)}$ Technical University \\ München; Germany
}

\begin{abstract}
Within the 'Bavarian Health Net' innovative applications will be combined using new communication techniques. This will lead to a standardized quality of medical services in all parts of Bavaria and can significantly reduce distance-induced costs and problems. It provides a basis for modern concepts such as shared care, distributed multi-media medical records, and protocol-based decision support.

Within the 'Intensive Medicine Framework' where high level medical computer science projects are founded, knowledge based documentation systems are integrated into the 'Bavarian Health Net'. Beside protocols and other techniques like Causal Probabilistic Networks (CPNs) also artificial neural networks (ANNs) are used to implement knowledge bases for complex decision tasks. In the medical domain there is often only a small number of examples available for the setup of a knowledge base and much care has to be taken of quality assurance. To meet these requirements the structured development method for supervised feed forward ANNs (SENN System Engineering for Neural Networks) that have to solve approximation or classification tasks, was developed.

The SENN method deals with the development of glass box systems which can be analysed and understood by an expert of the application domain. The development of adequate representations and encodings of the information, as well as the set-up, training, verification and validation of the ANN in a quality assured process are covered by the SENN method and are supported by the related tools.

Quality and efficiency of results obtained from evaluation studies in Diabetes care are promising for generating the ANN applicable for decision support. Further applications in the ByMediks ophthalmology subproject will show the usefulness of the approach, its possibilities and necessary enhancements on the way to a commonly tool.
\end{abstract}

Keywords

Medical Records, data analysis, neural networks, SENN method, development tool 


\section{INTRODUCTION}

Medical decisions are based on and documented in medical history and medical documentation plays a key role in medical data processing. It also links between all projects represented in the different health care areas of the Bavarian medical communication system (ByMediks). Data are collected problem- and treatment-oriented and are communicated between the different parties involved.

Medical decisions are also based on knowledge and experience which is documented in the different patients' medical records. There are several approaches to analyse the histories and extract medical knowledge using techniques such as case-based reasoning, statistical methods and explorative data analysis.

\section{BAVARIA ONLINE}

Under the heading "Bavaria Online“ the state of Bavaria has launched an initiative in telematics to show the benefit of this emerging technology starting with a limited number of application areas and a funding of 100 million DM.

One application area is medicine which has established the working party "Tele-Medicine". It has developed its own framework. Its main goal is to bridge the gap between high efficient health care, as e.g. provided in the university clinics, and the physician in a primary or secondary care environment. In the final stage also the patient shall be involved in a direct interactive way comparable to the "Bürger-Netz" a network that has already been set up for general applications open to all Bavarian inhabitants.

ByMediks (Bayerisches Medizinisches Informations- und Kommunikations System) is the working title for a project on a general clinical information and management system. It was thought more efficient to not start developing from scratch, but to adapt, complete and then reimplement existing solutions. The work plan for the next 2 years only focuses on few points and is based on projects running under the European telematic applications health framework (DIABCARD, GALEN in USE, and OPHTEL) and an online medical service HOS. It is planned to interlink all projects. This should not prove difficult because the medis-institute is engaged in all of these three projects and the service will be provided by a major publisher.

From the medical point of view the projects are somewhat interrelated

- ByMedCard deals with the development of a medical information system based on chip card technology in the area of chronic diseases with Diabetes mellitus as a paradigm;

- ByTelepräsenz focusses on clinical reporting and tele consulting using tele microscopy;

- ByOphtel provides knowledge for GPs and establishes the link between the GP and colleagues in tertiary care settings.

Two other projects, dealing with creating a public health network and medical protocols and guidelines fit into the scope of ByMediks and are planned to be included at a later stage.

\subsection{Horizontal layers}

For reasons of interoperability three pragmatic layers have been defined and groups have been established to work on medical documentation, terminology, processing algorithms, and technological aspects such as teleconsulting, networks, chipcards, data security, and privacy. 


\section{Medical documentation, terminology, patient cards}

Structured data input is the key requirement for an efficient communication between people and systems. This is based on a common set of terms and definitions as developed in projects such as EURODIABETA, DIABCARE, and DIABCARD for the field of Diabetes mellitus.

Technologies established in the GALEN and the DIADOQ projects will be used to develop and implement a problem and measure oriented, data driven and user adapted, resp. adaptable medical documentation system. Data base management and smart cards for data storage and communication will be essential parts. A commercially available system based on work of the NORA-project will be a major part of this concept.

\section{Tele consulting}

The consultation process is a key element in medical treatment and regularly takes place between a GP on the one side and medical specialists on the other side. Usually literature, specialists and experiences documented in case histories are consulted and several projects store this knowledge in knowledge bases. So consultation also means to get relevant information from knowledge bases in consultation mode.

\section{Networks, algorithms, health cards}

Network technology needs continous monitoring of tools and developments. LAN, Internet and Intranet technologies are used within the project. For security reasons smart cards for the health professionals will be introduced.

\subsection{Application areas}

The medical specialties which participate in ByMediks are endocrinology/diabetes mellitus, ophthalmology, surgery and pathology. There are commonalties between all application areas which ask for common approaches e.g. in medical knowledge based documentation.

The knowledge bases for the knowledge based documentation are realised with different approaches like protocols, Causal Probabilistic Networks (CPNs) and Artificial Neural Networks (ANNs). The knowledge bases of the 'Bavarian Health Net' can be accessed through the network and thus it cannot be guaranteed that only people who have passed a training process apply the offered tools and knowledge bases. Under these circumstances and especially in the medical domain restrictive quality assurance preconditions have to be fulfilled by the tools and knowledge bases. This makes it difficult to apply ANNs because, as often pointed out, the network structure of a ANN cannot be interpreted in a symbolic way. Another problem for the application of ANNs is the small number of examples that are typically available in the medical domain. To overcome these problems and to meet other strict requirements the structured development method for supervised feed forward ANNs SENN (System Engineering for Neural Networks) [Waschulzik 1993, Waschulzik 1995b] was developed and is applied in the 'Bavarian Health Net'.

\section{SENN}

ANNs are considered powerful tools when they are compared in scientific studies with statistical or machine learning algorithms [Michie 1994]. In recent years a lot of scientific progress has been made and the technology is ripe for wide-spread use in many areas. On the 
other hand problems may arise when ANNs are applied by general-purpose software engineers. One indicator for these problems is the large number of publications about systems with neural components compared to the small number of such systems that have been successfully evaluated in a prospective study [Waschulzik 1995a].

\subsection{Ten Problems in the Application of ANNs}

A development method that is typically used by engineers who are not involved in neural computing is described in [Maren 1990, Medsker 1994]. In this development method the less important information is eliminated while the more important information is enhanced in a pre-processing step. Afterwards one or more of the well-known network paradigms e.g. backpropagation [Rumelhart 1986], cascade correlation or RBF-networks are tested and the topologies and parameter settings are optimised. This procedure leads to the following problems which are also referenced in the literature:

1. Large example sets are necessary for the set-up of ANNs [Anthony 1994].

2. The training of ANNs is more CPU-consuming than statistical methods [Michie 1994].

3. The ANNs sometimes succeed in a given task on the training examples but fail on the test set [Anthony 1994].

4. In case of problem three it is difficult to detect the causes.

5. A ,glass-box test“ is impossible for many interesting network paradigms [Medsker 1994], which leads to problems in the verification of the network.

6. Many feedforward ANN paradigms suffer from parameters that have to be adapted empirically (e.g. the number of hidden neurons in a backpropagation network). The adjustment of these parameters is difficult because they have a non-linear influence on the quality of the ANN. [Caudill 1991].

7. The ANN fullfill their task at the training and test but fail in the field test. [Anthony 1994].

8. Existing theoretical knowledge about ANNs is often not applied.

9. The knowledge about proper parameter settings can not be reused in similar tasks.

10.According to the required man power the application of ANNs is not as efficient as the application of statistical or machine learning methods.

\subsection{The SENN Method}

The SENN method [Waschulzik 1993, Waschulzik 1995b] was developed to overcome these problems in applying feedforward ANNs with supervised learning to approximation and classification tasks. The SENN method is based on three basic concepts that differ from the generally used development method.

\subsubsection{Enhancement of the Similarities Between Input and Output}

OThe SENN method takes into account the fact that the networks to be constructed realise continuous mappings ${ }^{1}$ from the input to the output space, which leads to the following steps: The representation of the raw data has to be transformed in such a way that the function which has to be approximated by the ANN is smooth and has only small regions with large

\footnotetext{
${ }_{1}$ The SENN-Method uses neural networks that model continuous functions. The only exception is the final transformation of the network output in discrete values for integer outputs or for classification tasks.
} 
derivatives in the definition space. Such kind of functions support the possibility of the interpretation of the trained network and reduce the number of examples and the CPU-time required for the training of the ANN. In order to simplify the mapping from the input to the output space the input and output features have to be transformed. These transformations have to reduce the distances between those examples in the input space which have a similar output value as well as to enlarge the differences in the output space between those examples which do not have similar output values. The similarities between the input and the output space can be measured without training the ANN - in contrast to the importance of features. This process is called the enhancement of the similarities between the input and the output space. This is a very intuitive approach that can easily be explained to an expert of the application domain who can then propose adequate pre-processing steps. If the expert proposes different representations these can be evaluated by quality indicators which are based on the similarities in the input and the output space. Simple examples for quality indicators are the number of inconsistencies (the number of examples with the same input but a different output) and the equalities (the number of examples with the same representation in the input as well space as in the output space).

\subsubsection{Starting with the Simplest Adequate Encoding and Network}

In contrast to the philosophy behind RBF-networks, where the first layer can be seen as a special conjunctive encoding of the input variables, the SENN method separates the encoding phase from the network architecture used. Basic encodings used in the SENN method are e.g. the topological encoding [Geiger 1990] with an unsupervised adjustment of the positions of the neurons in the input space for numerical features and frequency dependent hierarchical encodings for hierarchical codes or mappings [Waschulzik 1993]. Before the information is fed into the ANN the basic encodings can be combined in order to simplify the mapping that has to be carried out by the ANN. One example for the combination of the basic encodings is the overlay encoding to represent a set of features. The basic encodings are combined by a commutative operator (usually + ) which throws away the sequence information. Another example for the combination of the encodings is the use of conjunctive encodings [Hinton 1986]. The quality of the encodings can be controlled by quality indicators. The development of the networks is started with the simplest adequate encodings, e.g. generally without the use of the conjunctive encoding which is often useless because it leads in most cases to larger networks and requires more examples than the parallel use of the basic encodings. The reduction of the dimensionality of the encoding reduces on the one side the capability of the network to learn complex mappings on the other side reduces the negative effect of the curse of dimensionality. The network structure used at the beginning of the development is a simple feedforward ANN with only an input and an output layer. This network structure can be used because the complexity of the task was reduced by the pre-processing and the selection of suitable encodings. If the set-up network is not sufficient for the given task, the complexity of the used encodings is enhanced, e.g. also conjunctive encodings are used. Thus a simple adequate network and encoding is found for a given task. If there are only numerical input features which are combined with conjunctive encoding, the resulting system consisting of the encoding and the network is equal to an RBF-network [Moody 1989]. 


\subsubsection{Support the User with Methods and Tools to Identify and Eliminate Reasons for Problematic Situations}

ANNs are often applied to tasks where an appropriate solution with conventional software techniques is not possible because an adequate formalization with conventional methods cannot be given. In this case a solution is only adequate if it can be realised, updated and maintained with appropriate resources. In solving the given task in such a difficult context it sometimes happens that a simulation result or a quality indicator signals failure of the actual approach. Adequate methods and tools in existing development methods fail to support the developer in these difficult situations. The SENN method, however, helps the developer with methods and tools to identify and eliminate the reasons for the failure of the actual approach. The ARE (Associative Recall of Examples) computes the difference between a given example and all other examples in the input space and presents the differences to the developer who can thus detect which examples most determine the output of the ANN for the actual example. The knowledge about the output of the examples in the neighbourhood of the input space from the example at hand helps the developer to improve the representations and encodings. The most important method in order to detect data collection artefacts and to investigate the functionality of the network is the analysis of the network structure. This is possible because simple network structures and encodings are used in the SENN method.

\subsection{Application of the SENN Method to the Ten Problems}

1. The size of the example sets can be reduced by integration of domain knowledge in the preprocessing step and avoiding the use of conjunctive encodings.

2. The training of a ANN set-up with the SENN method takes in general only some minutes on a general purpose workstation due to the simple network structure and is thus very fast compared to backpropagation networks [Förster 1995].

3. The generalisation of the network can be improved in a quality assured process guided by quality indicators.

4. The reasons for a wrong or insufficient generalisation of the network can be determined by the analysis of the network structure and the use of the ARE.

5. The simple network structure and encodings reduce the complexity and make a „glass-box test" possible.

6. The adjustment of the parameters for the networks used in the SENN method is much easier than for the commonly used feedforward networks, e.g. the number of hidden neurons in a backpropagation network [Waschulzik 1995b].

7. A failure of the network in the field test may be caused by two reasons: First, the ANN focused on data collection artefacts in the training and the test set which results in a low error rate in the test set. In the field test the information that was added by the data collection artefact is no more available and the network fails. Secondly, too many decisions made in the development process are based on the results of the test set and thus resul in an overadaption effect on the test set. This can be avoided by using an additional assessment set that had not been used before in the development process.

8. The SENN method is supported by the knowledge-based tool HEAD and the basic simulator PSIMUL. HEAD (Hybrid Expert system for the Analysis of Data) supports the user with theoretical knowledge about ANNs and proposes adequate encodings for the 
features. Another example for the support by HEAD is the analysis of the training and the test results in order to detect and avoid overadaption on the examples.

9. In contrast to the knowledge about good parameter settings for the training of a backpropagation network, the knowledge about adequate representations and encodings can be reused in the following project phases and also in similar projects.

10. When compared to a more conventional approach the SENN method proved very efficient compared to the use of backpropagation networks [Förster 1995].

\subsection{Approch of the SENN Method to the classification-problem ,secondary failure in type II diabetes"}

Type II Diabetes is characterised by an abnormal insulin secretion and a decreased insulin effect on insulin sensitive tissues (insulin resistance).

There is an urgent need for a reliable and real-time differential diagnosis in order to select and start an appropriate treatment assuring the best quality of life with the smallest degree of diabetes related complications [European NIDDM 1990].

The classification problem "secondary failure in type II Diabetes" (SF) was approached in two different ways to compare the proposed SENN method to a standard method.

It could be shown, that the conventional and the SENN-approach led to similar results after additional information on a proper encoding of the variables, on good combinations of data fields and on adequate network structures, all obtained by the SENN approach, was used in the conventional approach [Förster 1995]. Besides the effect on quality assurance which was proofed in an earlier medical project [Waschulzik 1993] where a complicated data collection artefact was detected by the SENN method an important advantage is the dramatically reduced time that was required for the development of the ANNs. It took 3 month using the conventional approach and 2 weeks using the SENN-Method .

\section{SUMMARY}

Efficiency and quality assurance in medical projects can be enhanced by using the SENN method and ANNs without hidden layers. The improved efficiency mainly depends on the approach of developing proper representations and encodings for the combination of variables instead of testing different network types (like cascade correlation or back propagation) with a time consuming parameter adjustment. The SENN-based development of ANNs can be better supported by tools because network parameters for the training of the perceptron can automatically be adjusted. Thus, the training and the test of networks can be conducted without any further manual interaction of the user. This also improves the quality of the project. The use of the representations and encodings for an associative recall of examples might also enable both an early evaluation of the first project phases as well as a context dependent analysis of the competence of the network.

Futer applications of the SENN Method are planed. One of these is the development of a ANN classifier for perimetry data as part of a system for knowledge based monitoring of glaucoma patients [Zahlmann]. First, rudimentary trials, using the SENN-Method showed promising results. 


\section{References}

Anthony D. (1994) Appropriate use of Neural Nets in Medical Imaging, Neural Networks and Expert Systems in Medicine and Healthcare, (eds. E. Ifeachor,K. Rosen), University of Plymouth, Plymouth, 45-52

Caudill M. (1991) Neural Network Training Tips and Techniques. AI Expert ,

Engelbrecht R., Rector A., Moser W. (1994) Verification and validation, in (ed. E.M.S.J. van Gennip, J.L. Talmon, Assessment and Evaluation of Information Technologies in Medicine . Amsterdam, IOS Press, 51-66

European NIDDM Policy Group (1990) A Desktop Guide for the Management of non-insulindependent diabetes mellitus. IDF Bulletin 25, 19901

Förster M, Kirchner K, Waschulzik T, et al (1994) DIADOQ: "Untersuchungen zum Aufbau von Wissensbasen in der Diabetologie mittels Neuronaler Netze". (Eds. H. Kunath et al.) Medizin und Information, MMV Medizin Verlag, München, 130-6

Geiger H. (1990) Storing and Processing Information in Connectionist Systems.- in (ed. R. Eckmiller) Advanced Neural Computers,. Elsevier (North Holland), Amsterdam, .271-7

Hinton G.E., McClelland J. L., Rummelhart D. E. (1986) Distributed Representations. in (eds. D. E. Rummelhart, J.L. McClelland and the PDP Research Group) Parallel Distributed Processing, MIT Press, Cambridge, MA 77-109

Maren A., Harston C. Pap R. (1990) Handbook of Neural Computing, Academic Press, Boston, New York, London

Medsker L., Libowitz, J (1994) Design and Development of Expert Systems and Neural Networks, Macmillan, New York, Toronto, Oxford, Singapore

Michie, D. (ed.) (1994) Machine Learning, Neural and Statistical Classification, Allis Horwood, New York,

Minsky M., Papert S. (1969) Perceptrons, MIT-Press Cambridge, MA:

Moody J., Darken C. (1989) Fast Learning in Networks of Locally-Tuned Processing Units, Neural Computation, Vol 1, 281-294

Rumelhart D. E., Hinton G. E., Williams R. J. (1986) Learning representations by backpropagating errors. Nature 323, 533-6

Waschulzik T. (1993) Evaluation of an Epidemiological Data Set as an Example of the Application of Neural Networks to the Analysis of Large Medical Data Sets. in: (eds Andreassen S., Engelbrecht R., Wyatt J.) Technology and Informatics 10, Artificial Intelligence in Medicine, IOS Press, Amsterdam 466-476.

Waschulzik T. (1995a) Neuronale Netze in der medizinischen Bildanalyse - Anwendungen und Aspekte der Qualitätssicherung und Qualitätskontrolle. in (Eds. L. DreschlerFischer, S. Pribbenow) KI-95, 19th Annual German Conference on Artificial Intelligence, Bielefeld,

Waschulzik T. et al. (1995b) Quality Assurance and Increased Efficiency in Medical Projects by Using a Structured Development Method for Feedforward Neural Networks (SENN). in: (Eds. Barahona, P. et al ) Artificial Intelligence in Medicine AIME '95. Lecture Notes in Artificial Intelligence. 934

Zahlmann G, M. Obermaier, C. Ritzke, M. (1996) Scherf Knowledge-based monitoring of glaucoma patients - a connectionist's approach, Proceedings MIE96, IOS Press, Amsterdam (in press) 